\title{
Linking a serotonin transporter polymorphism to vascular smooth muscle proliferation in patients with primary pulmonary hypertension
}

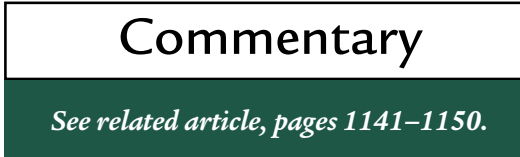

\begin{abstract}
Marlene Rabinovitch
Departments of Pediatrics, Laboratory Medicine and Pathobiology, and Medicine, University of Toronto, Cardiovascular Research Program, Research Institute, The Hospital for Sick Children, 555 University Avenue, 7003 McM, Toronto, Ontario, Canada M5G1X8. Phone: (416) 813-5918; Fax: (416) 813-7480; E-mail: mr@sickkids.on.ca.
\end{abstract}

J. Clin. Invest. 108:1109-1111 (2001). DOI:10.1172/JCI200114205.

Recent genetic studies in patients with familial and sporadic primary pulmonary hypertension $(\mathrm{PPH})$ have provided new opportunities to address the pathophysiologic mechanisms of this disease $(1,2)$. This past year, mutations in the bone morphogenetic protein receptor 2 (BMPR2) gene have been identified in even a higher proportion of individuals than was first appreciated (3). In addition, mutations associated with impaired signaling through other members of the TGF- $\beta$ receptor family have also been uncovered (4). How aberrant processing of these signals is related to the cellular mechanisms previously described in patients with pulmonary hypertension remains to be completely elucidated. In addition, we need to understand how other kinds of stimuli, such as inflammatory disorders associated with collagen vascular disease, mechanical forces related to congenital heart defects, and serotonin analogs like the appetite suppressant dexfenfluoramine, initiate cellular abnormalities that lead to severe progressive pulmonary hypertension.

\section{Serotonin and smooth muscle cell proliferation}

In this issue of the JCI, a novel study by Saadia Eddahibi et al. (5) from the laboratory of Serge Adnot helps to clarify a relationship between overexpression of the serotonin transporter (5-HTT) and the abnormally proliferative pulmonary artery smooth muscle cell phenotype in patients with PPH. The authors show that, compared with controls, patients with pulmonary hypertension more frequently carry the LL variant of
5-HTT, and that they show higher platelet uptake of serotonin (5-HT) and have higher levels of expression of 5-HTT mRNA and immunoreactivity in lung tissue. Moreover, pulmonary artery smooth muscle cells from patients with $\mathrm{PPH}$ show heightened uptake of 5-HT and expression of 5-HTT, and they proliferate more rapidly in response to $5-\mathrm{HT}$ - but not growth factors - than do cells from nonhypertensive individuals. Furthermore, cells from the subset of $\mathrm{PPH}$ patients with the LL polymorphism are even more highly proliferative in response to 5-HT than are cells from $\mathrm{PPH}$ patients who do not carry the polymorphism.

These studies follow from previous work by the same group (6-8), which showed that high levels of 5-HT accompany hypoxia-induced pulmonary hypertension and that antagonizing 5-HT can block the hypertensive response in rodents. The apparent link between 5-HT uptake and $\mathrm{PPH}$ is consistent with the authors' suggestion and their unpublished data indicating that appetite suppressant drugs not only serve as 5-HTT ligands but also stimulate 5-HTT expression.

Surprisingly, however, those patients in whom PPH can be attributed to ingestion of the appetite suppressant show the same proportion of 5-HTT polymorphism as do the $\mathrm{PPH}$ patients in general. One might have expected a higher incidence of the polymorphism in the group that ingested the appetite suppressants and developed disease. That is, despite the similar structure of 5-HT and the appetite suppressant compounds, there is little to explain why those patients without the poly- morphism develop disease and why there is a very high proportion of patients who have used these agents without developing pulmonary hypertension (9).

\section{Cross-talk with TGF- $\beta$ \\ signaling pathways}

It is not readily apparent why patients without the polymorphism show a higher than normal proliferative response to 5-HT. Perhaps undiscovered polymorphisms in other components of the 5-HT pathway, such as receptors or downstream effectors, also promote $\mathrm{PPH}$. In this regard, it would be of great interest to determine how the 5-HTT pathway is affected by a common BMPR2 mutation, which has been implicated in over $50 \%$ of individuals with familial and about $25 \%$ of those with sporadic $\mathrm{PPH}$. The authors allude to work in which normal signaling from BMPR2 is associated with suppression of smooth muscle cell proliferation (10, 11) and hence may antagonize the effects of 5-HT (Figure 1). The BMPR2 mutation could therefore allow for a heightened response to 5-HT, particularly in patients with a polymorphism allowing for heightened expression of the 5-HTT. To characterize the interaction between BMP and 5-HT signaling, it will be important to identify patients carrying both the 5HTT polymorphism and the BMPR2 mutation and to study the proliferative responses of their smooth muscle cells. This analysis could indicate how aberrant BMPR2 signal transduction and gene expression is complementary to increased 5-HTT signaling and gene expression in inducing cell prolifera- 


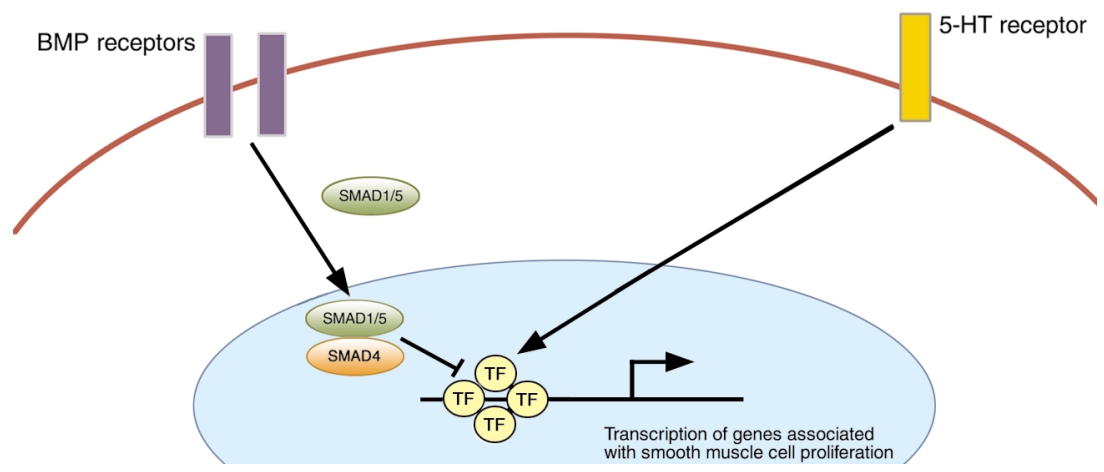

Figure 1

Hypothetical interaction between the pathways regulated by the bone morphogenetic protein receptor ligands and the serotonin (5-HT) transporter. Here, BMP receptor ligands are proposed to suppress transactivation of genes associated with smooth muscle proliferation by maintaining transcription factors (TF) bound to Smad proteins. Activation of the 5-HT transporter is normally antagonized by this pathway unless BMP receptor ligands are mutated or there is a polymorphism of 5-HT allowing for overexpression.

tion. For example, do the signals induced by 5 -HT and those suppressed by BMPR 2 ligands influence the transactivating ability of the same set of transcription factors and the expression of the same target genes?

In addressing the cellular biology of $\mathrm{PPH}$, the proliferative response of the pulmonary artery smooth muscle cell is only one facet of the disease. Indeed, despite high 5-HT levels and transport, hypoxia-induced pulmonary hypertension $(\mathrm{PH})$ is a reversible condition largely characterized by thickened vessels in which there are relatively modest cell proliferation and no increase in cell migration, as occurs in the characteristic obliterative lesions found in clinical PPH. Hence there may be other factors contributing to the disease process.

It has not been addressed how the proliferative response is related to other features of neointimal formation in advanced pulmonary vascular lesions, such as smooth muscle cell migration, the increased elaboration of ECM (12), or endothelial proliferation (13). For example, increased expression of growth factors, such as TGF- $\beta$, have been observed in patients (14) and in experimental models (15) of PH, and cells from patients with pulmonary hypertension are more highly responsive to the proliferative effect of TGF- $\beta$ (10). In addition, our group has shown in experimental animals that heightened serine elastase activity (16) liberates mitogenic growth factors from the ECM and promotes vascular smooth muscle cell proliferation (17).
Serine elastase can also initiate a proteolytic cascade that appears necessary for the induction of tenascin, a glycoprotein whose expression is also coupled to smooth muscle proliferation $(12,18,19)$. In cultured vascular smooth muscle cells, tenascin amplifies the proliferative response to growth factors by facilitating the phosphorylation of growth factor receptors (19).

It would also be of interest to explore polymorphism and heightened pulmonary hypertension in the fawn hooded rat $(20,21)$, which shows increased serotonin release and increased pulmonary hypertension in response to hypoxia but is free from other, more severe structural abnormalities. The role of serotonin in the pulmonary hypertensive phenotype has recently been called into question (21)

\section{Outlook}

The study by Eddahibi in this issue (5) provides the first clinical relationship between a genetic abnormality and a cellular process that is critical to the pathophysiology of pulmonary vascular disease. Its strength lies in the ability to use clinical tissue not only to make descriptive links but also to address biology. As such, this manuscript partially refutes widespread skepticism about cells maintaining phenotype in culture or being sufficiently representative of a complex process requiring a variety of cellular phenotypes (22). Like all important studies, however, it raises new questions that can only be addressed the relationship between the 5HTT with more extensive experiments. Greater in-depth analysis of the molecular mechanisms that ensue from heightened 5-HT transport will provide new insights and new targets to treat pulmonary vascular disease. Future studies may also address whether counteracting this mechanism leads not only to arrest in proliferation, but also to induction of apoptosis, as has been seen in experimental models with elastase inhibitors (23). New therapies that target 5-HT responses may be developed as adjuncts to or as replacements for intravenous prostacyclin, now the standard therapy for blocking the progression and inducing the regression of PPH $(24,25)$.

1. Deng, Z., et al. 2000. Familial primary pulmonary hypertension (gene PPH1) is caused by mutations in the bone morphogenetic protein receptor-Ii gene. Am. J. Hum. Genet. 67:737-744.

2. Lane, K.B., et al. 2000. Heterozygous germline mutations in BMPR2, encoding a TGF $\beta$ receptor, cause familial primary pulmonary hypertension. Nat. Genet. 26:81-84.

3. Newman, J.H., et al. 2001. Mutation in the gene for bone morphogenetic protein receptor II as a cause of primary pulmonary hypertension in a large kindred. N. Engl. J. Med. 345:319-324.

4. Trembath, R.C., et al. 2001. Clinical and molecular genetic features in pulmonary hypertension in patients with hereditary hemorrhagic telangiectasia. N. Engl. J. Med. 345:325-334.

5. Eddahibi, S., et al. 2001. Serotonin transporter overexpression in primary pulmonary hypertension contributes to arterial smooth muscle hyperplasia. J. Clin. Invest. 108:1141-1150.

6. Eddahibi, S., et al. 1997. Treatment with 5-HT potentiates development of pulmonary hypertension in chronically hypoxic rats. Am. J. Physiol. 272:H1173-H1181.

7. Eddahibbi, S., et al. 1999. Induction of serotonin transporter by hypoxia in pulmonary vascular smooth muscle cell. Relationship with the mitogenic action of serotonin. Circ. Res. 84:329-336.

8. Eddahibbi, S., et al. 2000. Attenuated hypoxic pulmonary hypertension in mice lacking the 5 hydroxytryptamine transporter gene. J. Clin. Invest. 105:1555-1562.

9. Abenheim, L., et al. 1996. Appetite-suppressant drugs and the risk of primary pulmonary hypertension. N. Engl. J. Med. 335:609-616.

10. Morrell, N.W., et al. 2001. Altered growth responses of pulmonary artery smooth muscle cells from patients with primary pulmonary hypertension to transforming growth factor beta(1) and bone morphogenetic proteins. Circulation. 104:790-795.

11. Doeai, H., Vukicevic, S., and Sampath, T.K. 2000 Bone morphogenetic protein -7 (osteogenic protein -1) inhibits smooth muscle cell proliferation and stimulates the expression of markers that are characteristic of SMC phenotype. J. Cell. Physiol. 184:37-45.

12.Jones, P.L., Cowan, K.N., and Rabinovitch, M 1997. Progressive pulmonary vascular disease is characterized by a proliferative response related to deposition of tenascin- $\mathrm{C}$ and is preceded by subendothelial accumulation of fibronectin. Am. J. Pathol. 150:1349-1360.

13. Lee, S.-D., et al. 1998. Monoclonal endothelial cell profileration is present in primary but not 
secondary hypertension. J. Clin. Invest. 101:927-934.

14. Botney, M.D., et al. 1993. Active collagen synthesis by pulmonary arteries in human primary pulmonary hypertension. Am. J. Pathol. 143:121-129.

15. Perkett, E.A., Pelton, R.W., Meyrick, B., Gold, L., and Miller, D.A. 1994. Expression of transforming growth factor $\beta$ mRNA and proteins in pulmonary vascular remodeling in the sheep with chronic pulmonary hypertension induced by air embolization. Am. J. Respir. Cell Mol. Biol. 11:16-24.

16. Ye, C.L., and Rabinovitch, M. 1991. Inhibition of elastolysis by SC-37698 reduces development and progression of monocrotaline pulmonary hypertension. Am. J. Physiol. 261:H1255-H1267.

17. Thompson, K., and Rabinovitch, M. 1996. Exogenous leukocyte elastase and endogenous elastases can mediate mitogenic activity in pulmonary artery smooth muscle cells by release of extracel lular matrix bound fibroblast growth factor. $J$ Cell. Physiol. 166:495-505.

18. Jones, P.L., and Rabinovitch, M. 1996. Tenascin$\mathrm{C}$ is induced with progressive pulmonary vascular disease in rats and is functionally related to increased smooth muscle cell proliferation. Circ. Res. 79:1131-1142.

19. Jones, P.L., Crack, J., and Rabinovitch, M. 1997. Regulation of tenascin-C, a vascular smooth muscle cell survival factor that interacts with the $\alpha v \beta 3$ integrin to promote epidermal growth factor receptor phosphorylation and growth. J. Cell Biol. 139:279-293.

20. Sato, K., et al. 1992. Factors influencing the idiopathic development of pulmonary hypertension in the fawn hooded rat. Am. Rev. Respir. Dis. 145:793-797.
21. Nagaoka, T., et al. 2001. Mild hypoxia causes severe pulmonary hypertension in fawn-hooded but not in Tester Moriyama rats. Respir. Physiol. 127:53-60.

22. Frid, M.G., Aldashev, A.A., Dempsey, E.C., and Stenmark, K.R. 1997. Smooth muscle cells isolated from discrete compartments of the mature vascular media exhibit unique phenotypes and distinct growth capabilities. Circ. Res. 81:940-952.

23. Cowan, K.N., Jones, P.L., and Rabinovitch, M 2000. Elastase and matrix metalloproteinase inhibitors induce regression and tenascin- $\mathrm{C}$ antisense prevents progression of vascular disease. $J$ Clin. Invest. 105:21-34.

24. Barst, R. 1998. Long term continuous prostacyclin reduces pulmonary vascular resistance in severe primary pulmonary hypertension. Clin. Exp. Rheumatol. 16:253-254. 